\title{
Morphological characterization of Aspergillus nidulans: growth, septation and fragmentation
}

\author{
Mhairi Mclntyre, Jens Dynesen and Jens Nielsen
}

Centre for Process

Biotechnology,

Department of

Biotechnology, Building

223, Technical University of Denmark, 2800 Lyngby, Denmark
Author for correspondence: Jens Nielsen. Tel: +454525 2694. Fax: +45 45884148. e-mail: jn@ibt.dtu.dk

The influence of the sepA gene on the growth of Aspergillus nidulans has been investigated by characterizing and comparing the parental strain A28 (pabaA6 biA1) with the sepA null mutant (sepA4 $\triangle B m$ ). The sepA gene is known to affect the septation process in $A$. nidulans, therefore the sepA4ABm strain does not produce any septa during the first hours of growth. During batch cultivations sepA4ABm shows an abrupt decrease in specific growth rate and more pronounced fragmentation (in response to elevated stirrer speed) than the parental strain. Higher specific fragmentation rates $\left(q_{\text {frag }}\right)$ were obtained for the sepA4ABm strain. The physiological reasons for the differences have been investigated by employing fluorescent stains. Computerized image analysis revealed that the more pronounced fragmentation in the mutant was due to the lower number and irregular spacing of septa (visualized by calcofluor white staining), which resulted in a weaker hyphal structure that is more vulnerable to shear stress and fragmentation than the parental strain. This led to a loss of active biomass (determined by Mag fura staining) from the hyphae of the mutant, which had failed to compartmentalize by formation of septa, in turn resulting in decreased specific growth rates for the culture.

Keywords: morphology, Aspergillus, septation, fragmentation, metabolic activity

\section{INTRODUCTION}

The morphology of filamentous fungi utilized in largescale submerged bioprocesses is a matter of considerable interest with regard to industrial process optimization. In addition to their well-documented roles in the production of primary and secondary metabolites, filamentous organisms are increasingly utilized as expression hosts for the production of heterologous proteins, where the yield of secreted protein is of primary interest. The morphology is assumed to affect productivity in two ways. Firstly, fungal macro- and micromorphology is known to affect the rheology of the fermentation medium (Bocking et al., 1999; Pedersen et al., 1993), thereby having a significant impact on the mixing, mass transfer and aeration within the bioreactor. This in turn may have an impact on protein production, which requires efficient mixing and aeration of the culture. Secondly, there have been a number of reports indicating that micro-morphology may influence productivity, with improved enzyme production being

Abbreviations: CFW, calcofluor white; DAPI, 4',6-diamidino-2-phenylindole dihydrochloride. observed in strains with altered morphologies (Bocking et al., 1999; Spohr et al., 1997). There has been particular focus on correlating the number of hyphal tips to protein secretion (Peberdy, 1994) as secretion has been shown to occur predominantly at the tips of filamentous fungi (Gordon et al., 2000; Torralba et al., 1998; Wessels, 1993; Wösten et al., 1991).

Elucidation of the physiological basis of the control of hyphal growth and branching is crucial if routes to the control and regulation of morphology on a molecular level are to be determined. It would be particularly desirable to identify the key genes influencing morphology in filamentous fungi as this would open the possibilities for mutant strains to be constructed with more desirable morphologies for submerged bioprocesses. Although attainment of such goals lies in the future, it is possible to facilitate this process through characterization of morphological mutants to determine the relationship between morphology, growth and behaviour of such strains in submerged cultivation.

A class of Aspergillus nidulans mutants defective in septation has been well described in the literature (Harris et al., 1994, 1997; Momany et al., 1995; Harris \& Hamer, 1995) and thus provides the strains required 
for characterization of defined morphological mutants in submerged cultivation. A sepA mutant $(\operatorname{sep} A 4 \Delta B m)$ is of particular interest; it is a null mutant that does not produce septa in the first hours of growth while displaying aberrant branching patterns and abnormally wide hyphae (Harris et al., 1997). Further, the positioning of nuclei in sepA deletion mutants is irregular; this is likely to be a result of lost control of hyphal compartment size due to the absence of septa.

Septation is an important event in the life cycle of filamentous fungi, compartmentalizing hyphal filaments as they are laid down, producing multi-nucleate 'cells'. Septum formation requires the integration of mitotic, cytokinetic and cell-wall biosynthetic events (Momany et al., 1995), enabling partitioning of the filaments in a regulated manner (following subsequent rounds of mitosis). Septation also allows differentiation of fungal surface colonies. Characteristic differentiation of cellular activities is exhibited along a hyphal filament depending on the location within a colony (Smith \& Anderson, 1973) and demonstrates the heterogeneous nature of the hyphal filament.

In A. nidulans, cytokinesis (septation) is initiated through the formation of a transient actin ring at the division site; a process that is closely co-ordinated with mitosis (Harris et al., 1997), ensuring chromosomes and organelles are properly partitioned in the resulting compartments (Momany et al., 1995). Septation requires nuclear division (Wolkow et al., 1996) and in A. nidulans septation is delayed until three rounds of nuclear division have concluded (Harris \& Kraus, 1998). Nuclear division occurs only in the apical compartment (Müller et al., 2000; King \& Alexander, 1969) with the onset of mitosis being regulated through a size mechanism relating the ratio of cytoplasmic volume to DNA (Trinci, 1979). In A. nidulans, size regulation is likely to be post-mitotic and the critical parameter controlling the onset of cytokinesis (Wolkow et al., 1996). The size (length) of the apical compartment increases with increasing specific growth rate (Müller et al., 2000). The subapical compartments of a mycelium, however, have a uniform size delimited by septa, and a regulated (average) number of nuclei (Müller et al., 2000; Wolkow et al., 1996).

Whilst previous studies of mutants defective in septation (Harris et al., 1994, 1997; Harris \& Hamer, 1995; Momany et al., 1995) or nuclear positioning (Wolkow et al., 1996; Xiang et al., 1994) have yielded valuable information on how the processes of cell growth, septation and mitosis are co-ordinated and regulated, the relevance of these phenotypes during submerged batch cultivations has not yet been examined.

The aim of this study, therefore, was to characterize the sepA deletion mutant of $A$. nidulans (sep $A 4 \Delta B m$ ) in submerged cultivation by comparison with the parental strain (A28). Batch cultivations were performed with high (600 r.p.m.) and low (300 r.p.m.) impeller speeds. As the sepA4ABm strain does not produce septa, this is likely to result in a weaker hyphal ultrastructure and the hyphae are, therefore, more likely to be susceptible to damage caused by shear stresses during cultivation in a bioreactor. Quantitative image analysis was applied to obtain values for the relative size of hyphal elements during cultivations and determine whether fragmentation was more pronounced in the septation deficient mutant. The specific fragmentation rate $\left(q_{\text {frag }}\right)$ was also estimated at different stages of growth to further investigate the differences between the two strains. The positioning of nuclei and septa (and thus, compartment lengths) were determined by employing computerized fluorescence image analysis with stains specific for the features of interest. In doing so, we have obtained data on the size of hyphal compartments and distribution of nuclei within both apical and subapical compartments. In addition to obtaining data on the macro- and micromorphology and the internal organization of hyphae, details of the physiological state of a culture are also of value. In particular, the level of metabolic activity within the culture can be determined by the use of fluorescent probes (Agger et al., 1998; Cox \& Thomas, 1999) and this adds further detail about the culture.

\section{METHODS}

Micro-organisms. Two strains of Aspergillus nidulans were used, a parental strain (A28; pabaA6 biA1) and a sepA deletion mutant $(\operatorname{sep} A 4 \Delta B m)$. The parental strain was obtained from the Fungal Genetics Stock Centre, Kansas City, KS, USA and the sepA $4 \Delta B m$ strain was kindly donated by S. D. Harris (Dept of Microbiology, University of Connecticut Health Center, Farmington, CT, USA). The organisms were cultivated in flasks containing $28 \mathrm{~g}$ rice, pre-autoclaved with $5 \mathrm{ml}$ medium (YPG $+p$-aminobenzioc acid), for $7 \mathrm{~d}$ at $30^{\circ} \mathrm{C}$. The YPG contained the following $\left(\mathrm{g} \mathrm{l}^{-1}\right)$ : yeast extract, 3; peptone, 10 and glucose, 20. The resulting spores were harvested in a $1 \%$ Tween solution and used to inoculate a fermenter vessel at $10^{5}$ spores $\mathrm{ml}^{-1}$.

Batch fermentation processes. Batch fermentations were carried out in a $5 \mathrm{l}$ (working volume) built-in-house fermentation vessel. The reactor consisted of a glass vessel with metal base and top plates through which were fitted a heater, temperature sensor and a cooling unit. The air supply, $\mathrm{pH}$ probe and $\mathrm{pH}$ control titrants were added through top plate ports. The aeration rate was 1 vol. air (culture vol. $)^{-1} \mathrm{~min}^{-1}$. The temperature was controlled at $30^{\circ} \mathrm{C}$ throughout. The $\mathrm{pH}$ for inoculation was $3 \cdot 0$, which was increased as the fermentation progressed and was then automatically controlled at $5 \cdot 0$ by addition of $1 \mathrm{M} \mathrm{NaOH}$. Stirrer speed in all processes was initially 100 r.p.m., which was increased as the fermentations progressed to either 300 or 600 r.p.m. The pH and stirrer speed were gradually increased over the time course of the processes to encourage filamentous growth.

The medium for batch fermentations contained $\left(\mathrm{g} \mathrm{l}^{-1}\right)$ : glucose, 40; $\left(\mathrm{NH}_{4}\right)_{2} \mathrm{SO}_{4}, 15 \cdot 0 ; \mathrm{KH}_{2} \mathrm{PO}_{4}, 3 \cdot 0 ; \mathrm{MgSO}_{4} .7 \mathrm{H}_{2} \mathrm{O}, 2 \cdot 0$; $\mathrm{NaCl}, 2 \cdot 0 ; \mathrm{CaCl}_{2} .2 \mathrm{H}_{2} \mathrm{O}, 0 \cdot 2$; and $1.0 \mathrm{ml} \mathrm{l}^{-1}$ trace element solution containing the following $\left(\mathrm{g} \mathrm{l}^{-1}\right): \mathrm{ZnSO}_{4} \cdot 7 \mathrm{H}_{2} \mathrm{O}, 14 \cdot 3$; $\mathrm{CuSO}_{4} .5 \mathrm{H}_{2} \mathrm{O}, 2 \cdot 5 ; \mathrm{NiCl}_{2} \cdot 6 \mathrm{H}_{2} \mathrm{O}, 0 \cdot 5$ and $\mathrm{FeSO}_{4} \cdot 7 \mathrm{H}_{2} \mathrm{O}, 13 \cdot 8$. Biotin and $p$-aminobenzioc acid were added aseptically after autoclaving to a final concentration of $1 \mathrm{mg} \mathrm{l}^{-1}$.

Morphological analysis. Morphological characteristics of the culture were measured using a Leica Quantimet 5501W 
computer with Leica Qwin software (both from Leica). The program produced digitized images from a Nikon microscope (Optiphot 2) on which was mounted a Kappa CF-8/1 FMC monochrome video camera (Kappa Messtechnik).

Mean area of hyphal elements. This was determined automatically using a QUIPS routine developed within the Leica Qwin software. Briefly, the threshold level was set on bright field images until the entire area of the hyphae to be measured was detected. The calibration was set such that the projected area $\left(\mu \mathrm{m}^{2}\right)$ of hyphal elements was calculated. The mean was computed from measurement of 100 hyphae. All samples were analysed at a total magnification of $160 \times$.

Mean compartment lengths of hyphal elements. The mean compartment lengths for hyphae were determined for the apical compartment, and first and second compartments distal to this. Lengths were obtained by skeletonizing hyphal trees and using a pre-set calibration to determine the distance between the apex and first septum (apical compartment) and between subsequent septa of each hyphal element (in $\mu \mathrm{m}$ ). Mean values were obtained from measurements of 100 hyphae. Samples were analysed at a total magnification of $400 \times$.

For all morphological analyses (including staining protocols), culture medium samples were first diluted to approximately $0.5 \mathrm{~g}$ biomass $\mathrm{l}^{-1}$. This prevented the occurrence of overlapping hyphae when the sample was placed on a glass microscope slide.

Estimation of the fragmentation rate. By assuming that fragmentation occurs with equal probability at any position in the hyphal elements and that the fragmentation function was proportional to the total hyphal length $l_{t}$, the specific fragmentation rate $q_{\mathrm{frag}}\left(l_{\mathrm{t}}, \boldsymbol{z}\right)\left(\mathrm{h}^{-1}\right)$ is given by

$q_{\mathrm{frag}}\left(l_{\mathrm{t}}, \boldsymbol{z}\right)=k_{\mathrm{frag}}(\boldsymbol{z}) \cdot l_{\mathrm{t}}$

(Nielsen \& Krabben, 1995) (where $k_{\text {frag }}(\boldsymbol{z})$ is a rate constant $\left(\mu \mathrm{m}^{-1} \mathrm{~h}^{-1}\right)$. When $q_{\mathrm{frag}}$ is given by (1) a balance for the mean total hyphal length $l_{\mathrm{t}, \mathrm{av}}$ can be expressed as

$\frac{d l_{\mathrm{t}, \mathrm{av}}}{d t}=n_{\mathrm{av}} \cdot q_{\mathrm{tip}}\left(l_{\mathrm{t}, \mathrm{av}}, \boldsymbol{z}\right)-q_{\mathrm{frag}}\left(l_{\mathrm{t}, \mathrm{av}}, \boldsymbol{z}\right) \cdot l_{\mathrm{t}, \mathrm{av}}$

(Nielsen \& Krabben, 1995) where $n_{\text {av }}$ is the mean number of actively growing tips and $q_{\mathrm{tip}}\left(l_{\mathrm{t}, \mathrm{av}}, \boldsymbol{z}\right)$ is the mean tip-extension rate in the hyphal elements $\left(\mathrm{m} \mathrm{tip}^{-1} \mathrm{~h}^{-1}\right)$. If the hyphal diameter is assumed to be constant $q_{\text {tip }}\left(l_{\mathrm{t}, \mathrm{av}}, z\right)$ is given by

$q_{\mathrm{tip}}\left(l_{\mathrm{t}, \mathrm{av}}, \boldsymbol{z}\right)=\mu(\boldsymbol{z}) \cdot \frac{l_{\mathrm{t}, \mathrm{av}}}{n_{\mathrm{av}}}$

which, by combination with (2) gives

$\frac{d l_{\mathrm{t}, \mathrm{av}}}{d t}=\mu(\boldsymbol{z}) \cdot l_{\mathrm{t}, \mathrm{av}}-q_{\mathrm{frag}}\left(l_{\mathrm{t}, \mathrm{av}}, \boldsymbol{z}\right) \cdot l_{\mathrm{t}, \mathrm{av}}$

Thus, the specific rate of fragmentation $q_{\text {frag }}\left(l_{\mathrm{t} \text {.av }}, \boldsymbol{z}\right)$ can be estimated using data on mean projected area of the hyphal elements (which is proportional to the hyphal length if the hyphal diameter is constant), and the specific growth rate $\mu(\boldsymbol{z})$. Since the observed specific growth rate is affected by fragmentation resulting in loss of biomass, the specific fragmentation rate may be underestimated. However, estimation of these rates still enables a quantitative comparison of this complicated process occurring in the different fermentations.

\section{Staining and fluorescent image analysis}

Calcofluor white (CFW). CFW (fluorescent brightener 28, Sigma; $100 \mu \mathrm{l})\left(0.3 \mathrm{~g} \mathrm{l}^{-1}\right)$ was added to $1 \mathrm{ml}$ diluted sample in a $1.5 \mathrm{ml}$ Eppendorf vial covered with tin foil. Samples were mixed and incubated at room temperature for $5 \mathrm{~min}$. A few drops of the solution were placed on a glass slide and covered with a coverslip for analysis. The dye fluoresces when bound to chitin and glucans, and thus stains cell walls and septa. Images were obtained using fluorescence microscopy on a Nikon Optiphot 2 microscope using a mercury burner and a filter block with $330-380 \mathrm{~nm}$ excitation wavelength and dichroic mirror with emission $>420 \mathrm{~nm}$.

The number of septa per area for each sample was obtained using a QUIPS routine written within Qwin software for image acquisition and analysis. Briefly, this involved automatic detection of the area of the hyphae (as above) using light microscopy. The same field of view was then captured using fluorescence microscopy where septa could be visualized, and the number within the hyphal area counted and entered manually in the QuiPs routine. The mean number of septa per area was determined from 100 fields of view for each sample, viewed at a total magnification of $400 \times$.

Mag fura. The Mag fura staining method reported by Cox \& Thomas (1999) was employed with minor modifications. Mag fura tetra potassium salt (Molecular Probes) was prepared in distilled water to a concentration of $0 \cdot 1 \mathrm{mg} \mathrm{ml}^{-1}$. Whole medium samples removed from batch fermentations were diluted to approximately $0.5 \mathrm{~g} \mathrm{l}^{-1}$ in buffer (citrate buffer, $\mathrm{pH} 5)$. Stain $(60 \mu \mathrm{l})$ was added to $1.5 \mathrm{ml}$ diluted sample, the mixture shaken for $5 \mathrm{~s}$ and allowed to stand for $2 \mathrm{~min}$. After this time the 'supernatant' was removed and the sample resuspended in an equal volume of buffer: this was necessary to reduce the background fluorescence of Mag fura, and thus obtain more accurate quantification of the area of stained hyphae. Slides were prepared by pipetting $40 \mu$ stained culture onto a microscope slide, covered with a coverslip. Samples were viewed under fluorescence microscopy using a mercury burner and a filter block with 330-380 nm excitation wavelength and dichroic mirror with emission $>420 \mathrm{~nm}$.

CFW with DAPI (4',6-diamidino-2-phenylindole dihydrochloride). The fluorochrome DAPI (Molecular Probes) was used to stain DNA in cell nuclei. Diluted sample $(60 \mu \mathrm{l})$ was added to $400 \mu \mathrm{l}$ PBS buffer $(\mathrm{pH} 9.4)$ in a $1.5 \mathrm{ml}$ Eppendorf vial coated with tin foil; to this $10 \mu \mathrm{l}$ DAPI $\left(2 \mu \mathrm{g} \mathrm{ml}^{-1}\right)$ and $50 \mu \mathrm{l} \mathrm{CFW}\left(0 \cdot 3 \mu \mathrm{g} \mathrm{ml}^{-1}\right)$ were added. The vial was shaken gently and allowed to incubate at room temperature for $10 \mathrm{~min}$. A few drops of the solution were then placed on a microscope slide, covered with a coverslip and the sample viewed under fluorescence microscopy as above.

\section{RESULTS AND DISCUSSION}

\section{Growth and micro-morphology}

The behaviour of two strains of A. nidulans was investigated in submerged batch cultivations operated at high and low stirrer speeds. In all processes (Fig. 1a-d), the stirrer speed was gradually increased over the lag and exponential phases of growth until the desired speed was attained and the resultant effect on specific growth rate determined. Whether final stirrer speed was low (300 r.p.m.; Fig. 1a, c) or high (600 r.p.m.; Fig. 1b, d), the sep $A$ deletion mutant exhibited a decrease in the net specific growth rate in response to increases in the stirrer 


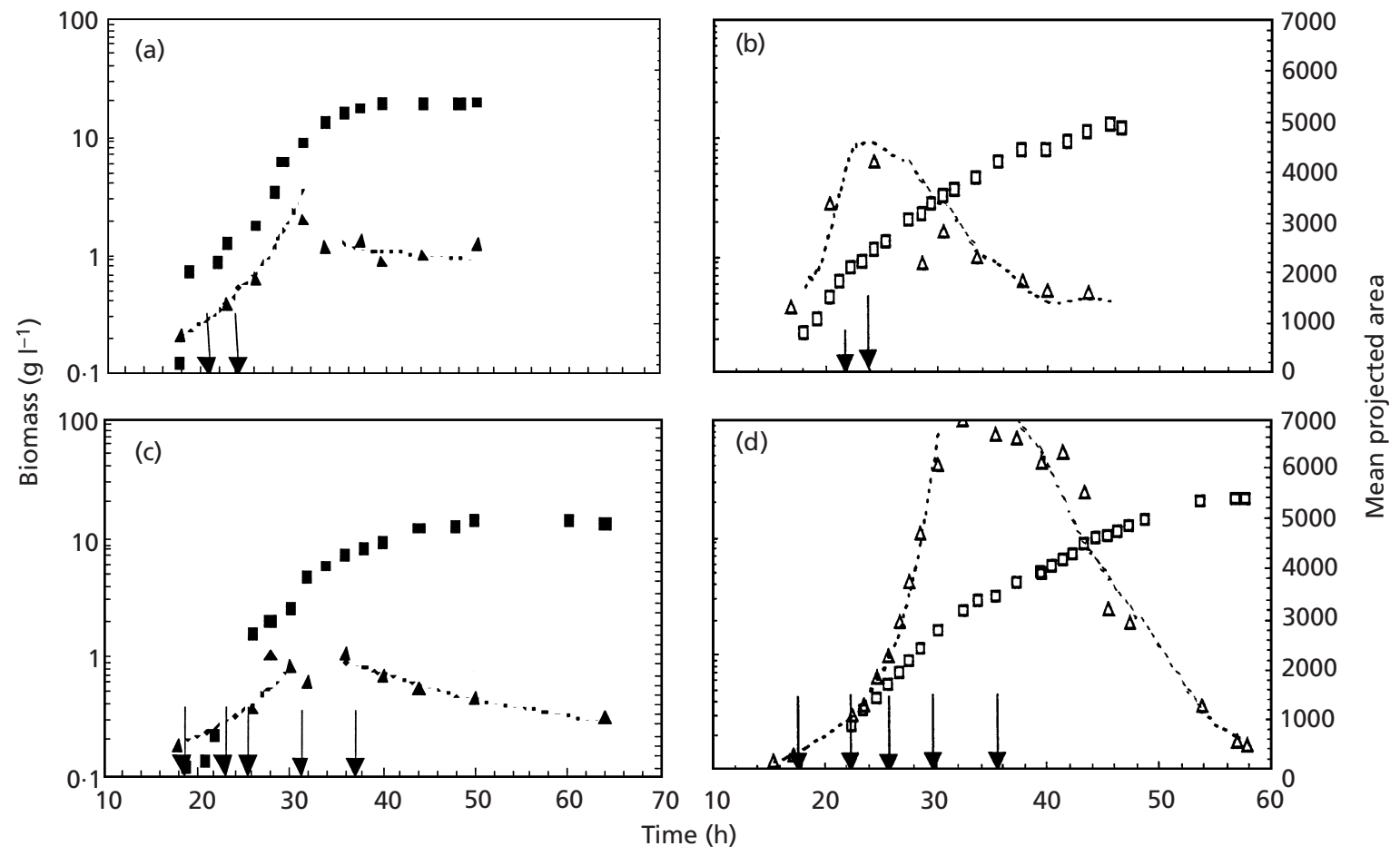

Fig. 1. Biomass concentration $(\boldsymbol{\square}, \square)$ and mean projected area of hyphal elements $(\boldsymbol{\Lambda}, \triangle)$. (a, c) A28 batch fermentation processes with final stirrer speed of 300 r.p.m. (a) or 600 r.p.m. (c). (b, d) sepA4 $\triangle B m$ batch fermentation processes with final stirrer speed of 300 r.p.m. (b) or 600 r.p.m. (d). Increases in stirrer speed are indicated by arrows. The lines represent modelled, mean projected area values for determination of the specific fragmentation rate $\left(q_{\text {frag }}\right)$.
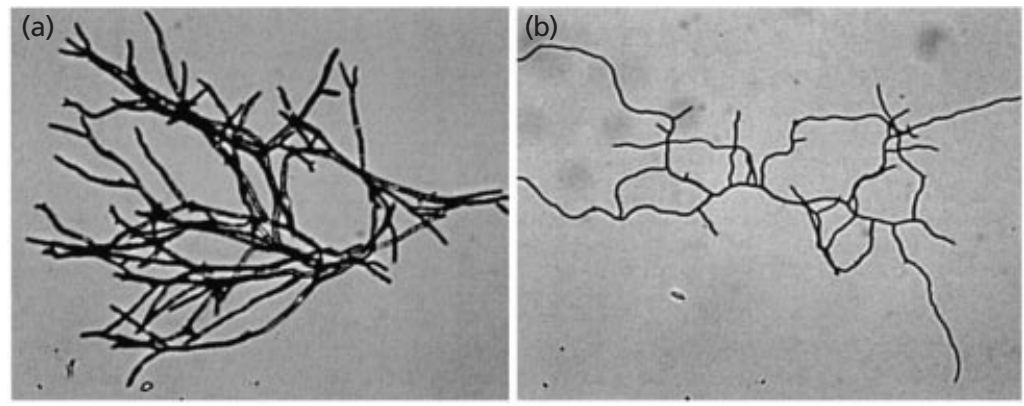

Fig. 2. Image analysis of hyphal elements. (a) Freely dispersed hyphal element of sepA4ABm from $30 \mathrm{~h}$ batch culture, stirrer speed 300 r.p.m. (b) Freely dispersed hyphal element of A28 from $30 \mathrm{~h}$ batch culture, stirrer speed 300 r.p.m. Both panels are the same magnification. speed, compared to the parental strain. Here the net specific growth rate is defined as the specific rate of hyphal growth minus the rate of hyphal death, due to fragmentation, that leads to loss of cell mass.

Morphological measurements of the culture were made in order to follow hyphal development and provide a primary means for comparison of the strains during the batch cultivations (Fig. 1). The microscopic morphology of filamentous fungi in submerged cultivation is determined by the growth kinetics of the hyphal element and the rate of fragmentation (Nielsen \& Krabben, 1995; Nielsen, 1993). It is of interest, therefore, to investigate these processes in the current study and determine their relative effects on morphology. From examination of Fig. 1, it is obvious that the maximum mean projected area of hyphae of the sep $A 4 \Delta B m$ strain is much larger than that obtained for the parental strain; the reason for this is illustrated in Fig. 2. The mutant strain produces hyphae that are wider than those of the parental strain and additionally has a tendency to form a large number of apical branches (Momany et al., 1995). This results in very dense structures of freely dispersed elements (arising from germination of a single spore) that do not form clumps (agglomerations of one or more free hyphal elements). Hyphae of strain A28, on the other hand, exhibit the classical hyphal tree structure, with narrower and less branched hyphae. The aberrant morphology of the mutant strain suggests a defect in cell wall biosynthesis or altered ultrastructural organization and this may provide an explanation for the decreased net specific growth rates observed in this culture at elevated stirrer speeds.

The sepA gene encodes a protein that may be capable of promoting formation of actin filaments (Harris et al., 
Table 1. Estimated fragmentation rate $\left(q_{\mathrm{frag}}\right)$ and specific growth rate $(\mu)$ for $A 28$ and sepA $A \triangle B m$ strains during the cultivations with low or high stirrer speeds

\begin{tabular}{|lcccc|}
\hline Strain & Stirrer speed & Time $(\mathbf{h})$ & $\boldsymbol{q}_{\text {frag }}\left(\mathbf{h}^{-1}\right)$ & $\boldsymbol{\mu}\left(\mathbf{h}^{-1}\right)$ \\
\hline \multirow{2}{*}{ A28 } & Low & $18-35$ & $0 \cdot 13$ & $0 \cdot 25$ \\
& & $35-60$ & $0 \cdot 01$ & $0 \cdot 02$ \\
& High & $18-30$ & $0 \cdot 24$ & $0 \cdot 32$ \\
& & $30-44$ & $0 \cdot 11$ & $0 \cdot 08$ \\
\multirow{3}{*}{ sepA44Bm } & $44-64$ & $0 \cdot 03$ & $0 \cdot 01$ \\
& Low & $15-23$ & $0 \cdot 08$ & $0 \cdot 31$ \\
& & $23-37$ & $0 \cdot 22$ & $0 \cdot 15$ \\
& High & $37-47$ & $0 \cdot 09$ & $0 \cdot 07$ \\
& & $15-30$ & $0 \cdot 01$ & $0 \cdot 24$ \\
& & $30-53$ & $0 \cdot 20$ & $0 \cdot 12$ \\
& & $53-55$ & $0 \cdot 20$ & $0 \cdot 01$ \\
\hline
\end{tabular}

1997). As actin influences hyphal architecture and polarity (Torralba et al., 1998; Bachewich \& Heath, 1998) any disruption to actin formation is likely to result in an aberrant morphology. sepA mutants have been shown to display defects in the establishment and maintenance of polarity (Harris et al., 1997). The increased hyphal width in the $\operatorname{sep} A 4 \Delta B m$ strain is assumed to be a result of the severe delay in the establishment of polarity (Harris et al., 1994, 1997), which may also be the cause of altered branching patterns.

In all processes, maximum mean projected hyphal area was attained towards the end of the phase of rapid exponential growth. Following this, the mean area decreased in all processes (Fig. 1). In the sep $A 4 \Delta B m$ strain, however, the decrease in area was far more pronounced than with the parental strain and was most marked in the process where the stirrer speed was high. Additionally, the maximum mean projected hyphal area reached in that cultivation (Fig. 1d) was lower than when the stirrer speed was low (Fig. 1b). This and the decline in mean hyphal area from the exponential growth phase onwards, suggests that gross fragmentation was occurring in the cultivations with the sep $A 4 \Delta B m$ strain.

The specific fragmentation rate was estimated using data obtained on the mean projected area of hyphal elements and the specific growth rates. The data are presented in Table 1. Early in the processes, the specific fragmentation rate was higher for the parental (A28) strain than the sepA4ABm strain. On average, sep $A 4 \Delta B m$ produces more germ tubes, which are also wider than those of the parental strain (data not shown). Therefore, for the same mean hyphal area in each strain, the mean area of each hypha was lower for the sep $A 4 \Delta B m$ strain. Thus, the specific fragmentation rate $\left(q_{\text {frag }}\right)$ was estimated to be lower for the sepA4ABm strain in the early stages of the fermentation. Later in the processes, the specific fragmentation rate of the sep $A 4 \Delta B m$ strain exceeds that of the parental strain, most likely due to the fact that the actin cytoskeleton is less organized in this strain (Harris et al., 1999) and septa (which normally provide a degree of rigidity) are lacking.

When specific fragmentation rates and specific growth rates for the strains are compared under different conditions, it can be seen that fragmentation results in a reduction in the net specific growth rate for the sepA $4 \Delta B m$ strain. For the A28 (parental) strain, the last value in each set (Table 1) represents the culture in the stationary phase (the specific growth rate is low). However, the specific fragmentation rate is also low in these cases, despite the fact that the strain is exposed to the effects of impeller-induced shearing. The specific fragmentation rate is only slightly higher in the process with the high stirrer speed $(0.03$ compared to 0.01 at the low stirrer speed). For the sep $A 4 \Delta B m$ strain, however, fragmentation was observed throughout the process, with a higher specific fragmentation rate being calculated in the process where the stirrer speed was high.

\section{Physiological investigations}

The internal organization of the hyphae was investigated by employing specific fluorescent stains and computerized image analysis. It is known that the $s e p A 4 \Delta B m$ strain is deficient in producing septa, at least in the first hours of growth (Momany et al., 1995).

CFW is a stilbene compound which binds to glucans and chitin in the cell walls and septa of fungi (Agger et al., 1998; Kasten, 1993), and was used in the current study to aid visualization of septa in hyphal samples from batch fermentation processes. The mean projected area of hyphae per septum was calculated for each of the cultivations at a number of time points during the time course. The results are shown in Fig. 3.

For the parental strain, in both cultivations, the maximum area per septum was around $2000 \mu \mathrm{m}^{2}$, which occurred during the exponential phase of growth. After this time the area per septum declined to a final minimum value of around $1000 \mu \mathrm{m}^{2}$ in both cultivations. 


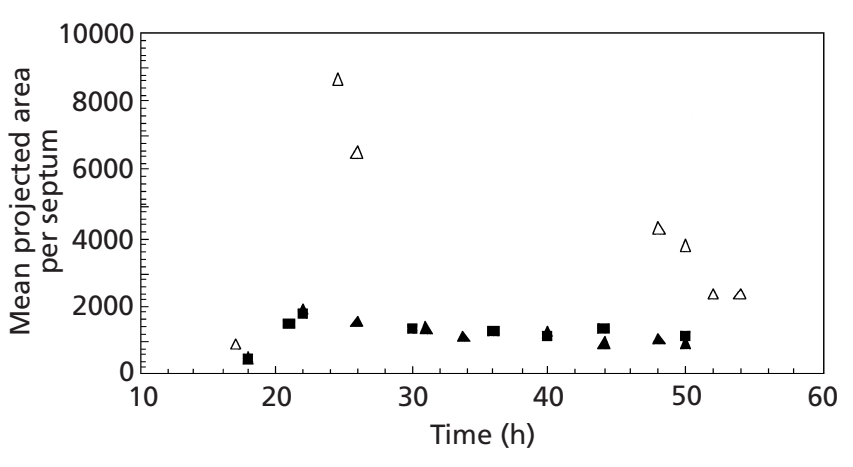

Fig. 3. Number of septa per area for $A 28$ at low $(\boldsymbol{A})$ and high $(\boldsymbol{D})$ stirrer speeds, and $\operatorname{sep} A 4 \triangle B m$ at low $(\triangle)$ and high $(\square)$ stirrer speeds during batch cultivations.

As expected, the area per septum for the mutant strain was much higher than for the parental strain, reaching approximately $7000 \mu \mathrm{m}^{2}$ during the exponential phase of growth in the cultivation with low stirrer speed. In this study, the first septa in the sep $A 4 \Delta B m$ strain were observed at around $16 \mathrm{~h}$; however this is dependent on the process conditions and, therefore, the time for appearance of the first septum has been shown to vary between studies.

To explain the observations of Fig. 3, the processes of growth and fragmentation should be considered as these influence the micro-morphology of the culture (Nielsen \& Krabben, 1995). It is reasonable that the maximum area of hyphae supported per septum occurs when the specific growth rate is highest as the apical compartment length has been shown to increase as the specific growth rate increases (Müller et al., 2000). This may account for the initial rise in area per septum during the exponential growth phase. The area per septum then decreases over the remainder of the time course (Fig. 3) and this could be due to two distinct processes - septation or fragmentation. The most obvious explanation is that more septa would have been produced later in the fermentations, and this coupled with the fact that apical compartment size decreases with a decline in growth rate would have resulted in a lower area per septa being calculated. It is known that the $\operatorname{sep} A 4 \Delta B m$ strain produces septa later in batch cultivations; however, based on our observations, the number of new septa was low and this alone would not have been sufficient to account for the decreases observed. Additionally, based on results from previous studies (Müller et al., 2000), the change in the length of the apical compartment could not have resulted in the dramatic decline in area per septum observed in this study. We have already discussed the fact that fragmentation occurs in all cultures during the stationary phase of growth and it is, therefore more likely that this process contributed to the observed decline in the area per septum.

In filamentous fungi, the duplication cycle involves the integration of tip growth, nuclear division, septation and branching (Trinci, 1978). When the nuclei have duplicated, they separate and the cycle ends with the laying down of a septum that results in the formation of a new apical compartment (Trinci, 1979). Tip extension rates (i.e. hyphal duplication) have been shown to increase as the specific growth rate increases (Spohr et al., 1997; Nielsen \& Krabben, 1995). Information about how a filamentous fungus septates and controls the duplication cycle may be obtained by measurement of the compartment lengths in the hyphae (Müller et al., 2000). This is of particular interest in the context of the study presented here, where the $\operatorname{sep} A 4 \Delta B m$ strain is under investigation. Additionally, septation is an important event in the differentiation of fungal hyphae, resulting in compartmentalization of hyphal sections and cellular functions (Smith \& Anderson, 1973). This emphasizes the important role of septation in the life cycle of filamentous fungi, both during their growth in nature and when cultivated in bioreactors.

The lengths of the apical and subapical compartments of the hyphae of both the parental strain and sepA $4 \Delta B m$ strains, under both sets of cultivation conditions, are given in Table 2. Apical compartment length has been shown to increase with increasing specific growth rate in Aspergillus oryzae and Aspergillus niger (Müller et al., 2000) and in the work presented here, apical compartment length was consistently higher during the

Table 2. Length of compartments ( $\mu \mathrm{m} \pm \mathrm{SD}$ ) of $\mathrm{A} 28$ and $\operatorname{sep} A 4 \Delta B m$ hyphae during batch cultivations at low $(\mathrm{L})$ and high $(\mathrm{H})$ stirrer speed

\begin{tabular}{|lcccc|}
\hline Strain & $\begin{array}{c}\text { Sample } \\
\text { time (h) }\end{array}$ & $\begin{array}{c}\text { Apical } \\
\text { compartment }\end{array}$ & $\begin{array}{c}\text { First } \\
\text { compartment }\end{array}$ & $\begin{array}{c}\text { Second } \\
\text { compartment }\end{array}$ \\
\hline A28 & $26(\mathrm{~L})$ & $176 \pm 42$ & $33 \pm 13$ & $24 \pm 6$ \\
& $50(\mathrm{~L})$ & $50 \pm 12$ & $19 \pm 6$ & $17 \pm 4$ \\
& $26(\mathrm{H})$ & $185 \pm 31$ & $39 \pm 14$ & $37 \pm 7$ \\
sepA4ABm & $50(\mathrm{H})$ & $18 \pm 9$ & $15 \pm 5$ & - \\
& $24(\mathrm{~L})$ & $266 \pm 81$ & - & - \\
& $44(\mathrm{~L})$ & $251 \pm 75$ & $160 \pm 79$ & - \\
& $24(\mathrm{H})$ & $443 \pm 103$ & - & - \\
\hline
\end{tabular}


Table 3. Mean number of nuclei per compartment of $A 28$ and sepA4ABm hyphae during batch cultivations at low $(\mathrm{L})$ and high $(\mathrm{H})$ stirrer speeds

\begin{tabular}{|lcccc|}
\hline Strain & $\begin{array}{c}\text { Sample } \\
\text { time (h) }\end{array}$ & $\begin{array}{c}\text { Apical } \\
\text { compartment }\end{array}$ & $\begin{array}{c}\text { First } \\
\text { compartment }\end{array}$ & $\begin{array}{c}\text { Second } \\
\text { compartment }\end{array}$ \\
\hline \multirow{2}{*}{ A28 } & $26(\mathrm{~L})$ & $10 \cdot 8$ & $3 \cdot 8$ & $3 \cdot 1$ \\
& $50(\mathrm{~L})$ & $6 \cdot 3$ & $2 \cdot 9$ & $2 \cdot 7$ \\
& $26(\mathrm{H})$ & $12 \cdot 5$ & $3 \cdot 5$ & $2 \cdot 5$ \\
sepA44Bm & $50(\mathrm{H})$ & $2 \cdot 7$ & $2 \cdot 6$ & $2 \cdot 4$ \\
& $24(\mathrm{~L})$ & $61 \cdot 5$ & - & - \\
& $44(\mathrm{~L})$ & $55 \cdot 2$ & $34 \cdot 7$ & - \\
& $24(\mathrm{H})$ & $58 \cdot 6$ & - & - \\
\hline
\end{tabular}

exponential phase of growth ( 26 or $24 \mathrm{~h}$ sample) than during the stationary growth phase. The length of the apical compartments was much higher for the sep $A 4 \Delta B m$ strain; most hyphae consisted of only one compartment as less septa are formed in this strain. For both strains, apical compartment lengths were lowest during stationary phase when stirrer speed was high; this was likely to be due to the lower specific growth rate (Müller et al., 2000).

The number of nuclei in the hyphal compartments was calculated after DAPI and CFW staining of hyphae. The results are given in Table 3 . The number of nuclei per compartment was consistently higher for the sepA $4 \Delta B m$ strain than the parental strain. This was not only due to the absence of septa in the sep $A 4 \Delta B m$ strain; a mean length of $9.9 \mu \mathrm{m}$ per nucleus was calculated for the parental strain (A28) and $5.8 \mu \mathrm{m}$ for the sepA4ABm strain. Additionally, the nuclei were not regularly spaced in the sep $A 4 \triangle B m$ strain, as was observed with the parental strain (A28). It has been shown that SEPA (the protein product of the sepA gene) is needed to efficiently organize the actin cytoskeleton (Harris et al., 1999) in A. nidulans. Spatially and temporally controlled nuclear migration in filamentous fungi requires the co-ordinated involvement of cytoskeleton components, motor components and regulatory proteins (Fischer, 1999). Therefore, it is reasonable that an uneven distribution of nuclei would be observed in the sep $A 4 \Delta B m$ strain.

The relationship between specific growth rate, septation and fragmentation was further investigated by determining the physiological state of hyphae during each of the batch cultivations. Previously, fluorescent stains have been used to determine the level of metabolic activity within fungal cultures (Cox \& Thomas, 1999; Agger et al., 1998) with a view to measuring and modelling hyphal differentiation. Cox \& Thomas (1999) developed a new staining method to determine metabolic activity in fungal hyphae based on fluorescence activity of Mag fura. Fluorescence was associated with active regions in fungal hyphae, with a positive response being a bright emission close to the cell membrane. In regions without the high response, a weak emission at the periphery of the cell wall only is observed. It was
Table 4. Activity in batch cultures of A28 and sep $A 4 \Delta B m$ where stirrer speed was increased to 600 r.p.m., as determined by Mag fura (for active membranes)

\begin{tabular}{|lcc|}
\hline $\begin{array}{l}\text { Process } \\
\text { time }(\mathbf{h})\end{array}$ & $\begin{array}{c}\text { Activity Mag } \\
\text { fura A28 (\%) }\end{array}$ & $\begin{array}{c}\text { Activity Mag fura } \\
\boldsymbol{s e p} \boldsymbol{A 4 \Delta B \boldsymbol { M } ( \% )}\end{array}$ \\
\hline 18 & 77 & 73 \\
24 & 75 & 51 \\
42 & 58 & 32 \\
\hline
\end{tabular}

hypothesized that the stain was responding to a localized flux of divalent cations from the cell membrane and was thus staining membranes with high ATPase activity. Staining of the mitochondria is an alternative method for determining the metabolic activity of hyphae. Agger et al. (1998) calculated the ratio between the projected area of stained (fluorescent) mitochondria and of the entire hyphal element and assumed this was proportional to the fraction of active cells. Coupling fluorescence detection and image analysis, it was possible, therefore, in the current study to calculate the percentage of active hyphae within a sample; in this paper the Mag fura staining method was employed. The results are presented in Table 4.

In our study, cultures were most active during the exponential phase of growth $(18 \mathrm{~h})$, with metabolic activity decreasing as the fermentations proceeded. The culture with the parental strain lost little activity relative to the $\operatorname{sep} A 4 \Delta B$ strain but it appears that both cultures displayed a reduced activity, concomitant with a decline in specific growth rate, as stirrer speed was increased. The effect was most pronounced in the $\operatorname{sep} A 4 \Delta B$ cultures. From analysis of the morphological data, the main difference between the two strains in submerged cultivation was that $\operatorname{sep} A 4 \Delta B$ decreased in size from the end of the exponential phase of growth onwards. This was due to gross fragmentation in response to elevated stirrer speeds. When the physiological evidence is considered, it appears that the fragmentation resulted in a loss of metabolic activity from sep $A 4 \Delta B$. Taking into 
account the number of septa in the hyphae of this strain, a reason for the above observations can be postulated. The hyphal elements of $\operatorname{sep} A 4 \Delta B$ are weaker than the parental strain due to the absence of septa and altered ultrastructural organization. This means that fragmentation results when shear forces are high. Because there is less compartmentalization in this strain (which is normally provided through septation), a large amount of active cytoplasm is lost on fragmentation. This led to a lowering in the net specific growth rate and overall metabolic activity for the culture. In the parental strain, the presence of septa prevented such gross loss of cytoplasm on fragmentation and this culture was able to maintain a higher level of metabolic activity while the stirrer speed was being increased.

\section{ACKNOWLEDGEMENTS}

The research on Aspergillus at the Center for Process Biotechnology is supported by Novo Nordisk A/S.

\section{REFERENCES}

Agger, T., Spohr, A. B., Carlsen, M. \& Nielsen, J. (1998). Growth and product formation of Aspergillus oryzae during submerged cultivations: verification of a morphologically structured model using fluorescent probes. Biotechnol Bioeng 57, 321-329.

Bachewich, C. \& Heath, I. B. (1998). Radial F-actin arrays precede new hypha formation in Saprolegnia: implications for establishing polar growth and regulating tip morphogenesis. J Cell Sci 111, 2005-2016.

Bocking, S. P., Wiebe, M. G., Robson, G. D., Hansen, K., Christiansen, L. H. \& Trinci, A. P. J. (1999). Effect of branch frequency in Aspergillus oryzae on protein secretion and culture viscosity. Biotechnol Bioeng 65, 638-648.

Cox, P. W. \& Thomas, C. R. (1999). Assessment of the activity of filamentous fungi using Mag fura. Mycol Res 103, 757-763.

Fischer, R. (1999). Nuclear movement in filamentous fungi. FEMS Microbiol Rev 23, 39-68.

Gordon, C. L., Khalaj, V., Ram, A. F. J. \& 9 other authors (2000). Glucoamylase::green fluorescent protein fusions to monitor protein secretion in Aspergillus niger. Microbiology 146, 415-426.

Harris, S. D. \& Hamer, J. E. (1995). sepB: an Aspergillus nidulans gene involved in chromosome segregation and the initiation of cytokinesis. EMBO J 14, 5244-5257.

Harris, S. D. \& Kraus, P. K. (1998). Regulation of septum formation in Aspergillus nidulans by a DNA damage checkpoint pathway. Genetics 148, 1055-1067.

Harris, S. D., Morrell, J. L. \& Hamer, J. E. (1994). Identification and characterization of Aspergillus nidulans mutants defective in cytokinesis. Genetics 136, 517-532.

Harris, S. D., Hamer, L., Sharpless, K. E. \& Hamer, J. E. (1997). The Aspergillus nidulans sepA gene encodes an $\mathrm{FH} 1 / 2$ protein involved in cytokinesis and the maintenance of cellular polarity. EMBO J 16, 3474-3483.

Harris, S. D., Hofmann, A. F., Tedford, H. W. \& Lee, M. P. (1999). Identification and characterization of genes required for hyphal morphogenesis in the filamentous fungus Aspergillus nidulans. Genetics 151, 1015-1025.
Kasten, F. H. (1993). Introduction to fluorescent probes. In Fluorescent and Luminescent Probes for Biological Activity, pp. 12-33. Edited by W. T. Mason. London: Academic Press.

King, S. B. \& Alexander, L. J. (1969). Nuclear behaviour, septation and hyphal growth of Alternaria solani. Am J Bot 56, 249-253.

Momany, M., Morrell, J. L., Harris, S. D. \& Hamer, J. E. (1995). Septum formation in Aspergillus nidulans. Can J Bot $\mathbf{7 3}$ (Suppl. 1), S396-S399.

Müller, C., Spohr, A. B. \& Nielsen, J. (2000). Role of substrate concentration in mitosis and hyphal extension of Aspergillus. Biotechnol Bioeng 67, 390-397.

Nielsen, J. (1993). A simple morphologically structured model describing the growth of filamentous micro-organisms. Biotechnol Bioeng 41, 715-727.

Nielsen, J. \& Krabben, P. (1995). Hyphal growth and fragmentation of Penicillium chrysogenum in submerged cultures. Biotechnol Bioeng 46, 588-598.

Peberdy, J. F. (1994). Protein secretion in filamentous fungi trying to understand a highly productive black box. Trends Biotechnol 12, 50-57.

Pedersen, A. G., Bundgaard-Nielsen, M., Nielsen, J., Villadsen, J. \& Hassager, O. (1993). Rheological characterisation of media containing Penicillium chrysogenum. Biotechnol Bioeng 41, 162-164.

Smith, J. E. \& Anderson, J. G. (1973). Differentiation in the Aspergilli. In Microbial Differentiation, 23rd Symposium of the Society for General Microbiology, pp. 295-336. Edited by J. M. Ashworth and J. E. Smith. Cambridge: Cambridge University Press.

Spohr, A., Carlsen, M., Nielsen, J. \& Villadsen, J. (1997). Morphological characterisation of recombinant strains of Aspergillus oryzae producing $\alpha$-amylase during batch cultivations. Biotechnol Lett 19, 257-261.

Trinci, A. P. J. (1978). The duplication cycle and vegetative development in moulds. In The Filamentous Fungi, vol. III, pp. 132-163. Edited by J. E. Smith and D. R. Berry. London: Edward Arnold.

Trinci, A. P. J. (1979). The duplication cycle and branching in fungi. In Fungal Walls and Hyphal Growth, pp. 319-358. Edited by J. H. Burnett and A. P. J. Trinci. Cambridge: Cambridge University Press.

Torralba, S., Raudaskoski, M., Pedregosa, A. M. \& Laborda, F. (1998). Effect of cytocholasin A on apical growth, actin cytoskeleton organization and enzyme secretion in Aspergillus nidulans. Microbiology 144, 45-53.

Wessels, J. G. H. (1993). Wall growth, protein excretion and morphogenesis in fungi. New Phytol 123, 397-413.

Wolkow, T. D., Harris, S. D. \& Hamer, J. E. (1996). Cytokinesis in Aspergillus nidulans is controlled by cell size, nuclear positioning and mitosis. J Cell Sci 109, 2179-2188.

Wösten, H. A. B., Moukha, S. M., Sietsma, J. H. \& Wessels, J. G. H. (1991). Localisation of growth and secretion of proteins in Aspergillus niger. J Gen Microbiol 137, 2017-2023.

Xiang, X., Beckwith, S. M. \& Morris, N. R. (1994). Cytoplasmic dynein is involved in nuclear migration in Aspergillus nidulans. Proc Natl Acad Sci U S A 91, 2100-2104.

Received 9 June 2000; revised 15 August 2000; accepted 25 September 2000. 